\title{
Histological and antimicrobial effects of tramadol infiltration on incisional oral mucosal wound healing in rabbits
}

\author{
F.A. Al-Mashhadane, E.A. Mustafa and G.A. Taqa \\ Department of Basic Science, College of Dentistry, University of Mosul, Mosul, Iraq
}

(Received November 11, 2018; Accepted January 12, 2019)

\begin{abstract}
The present study compared effects of tramadol with lidocaine on healing processes of incisional intraoral mucosal wounds in rabbits and to study the antimicrobial effect of tramadol. For this purpose, fifteen apparently healthy male rabbits of 5-6 months old were used. They were classified into three groups, first group treated with $0.5 \mathrm{ml}$ distilled water infiltration as control ( $\mathrm{n}=5)$, second group treated with $0.5 \mathrm{ml}$ tramadol infiltration in dose of $100 \mathrm{mg} / 2 \mathrm{ml}(\mathrm{n}=5)$, and third group treated with $0.5 \mathrm{ml}$ of $2 \%$ vasoconstrictor-free lidocaine infiltration, then an incision was done to the buccal mucosa of all animals and they slaughtered on day 10 and their wounds were evaluated by histopathologic for presence of edema, vascularity, and inflammatory cells. For antimicrobial effect, swabs from tramadol group were taken from sites of wounds before incision and other swabs were taken after surgical incision on the first day, third day and seventh day, then the colony forming units $(\mathrm{CFU}) / \mathrm{ml}$ of microorganisms were counted. The present study found that the infiltration with tramadol in dose of $100 \mathrm{mg} / 2 \mathrm{ml}$ result in new epithelialization and wound healing occurred faster than infiltration with lidocaine $2 \%$ group also this study found that tramadol has significant decrease in the means of colony forming units of oral microorganisms. This study demonstrated that tramadol accelerated stages of buccal wound healing faster than lidocaine in rabbits.
\end{abstract}

Keywords: Tramadol, Oral mucosa, Wound healing, Antimicrobial effect Available online at http://www.vetmedmosul.com

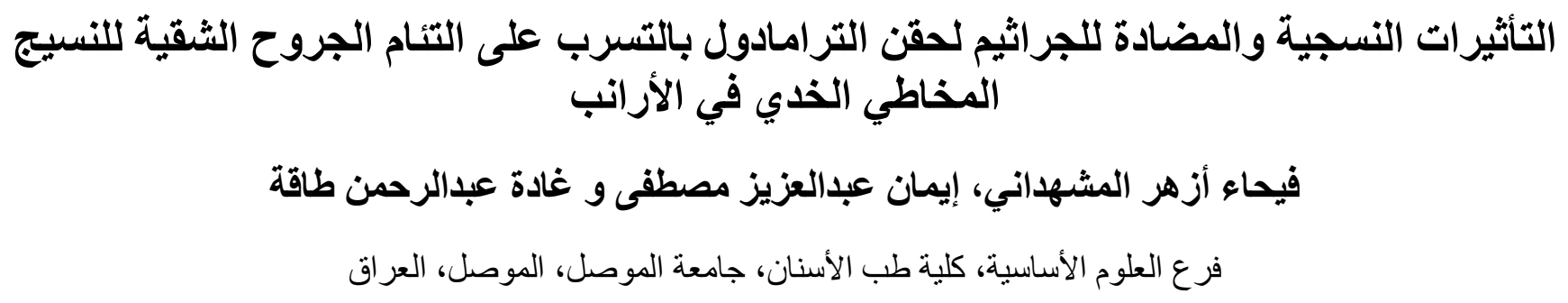

الخلاصة

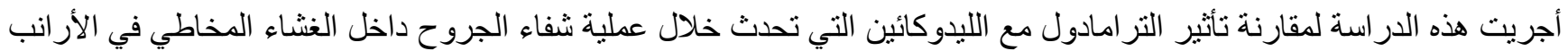

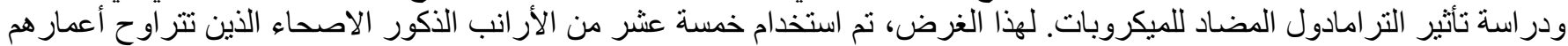

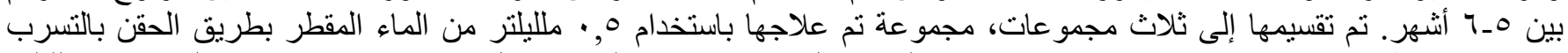

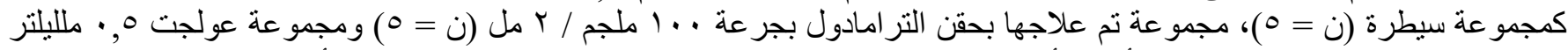

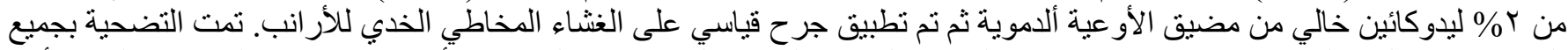

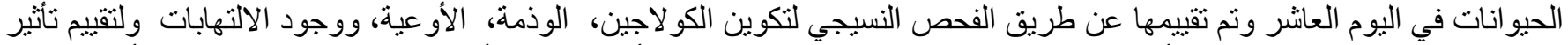

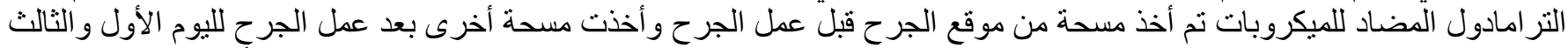

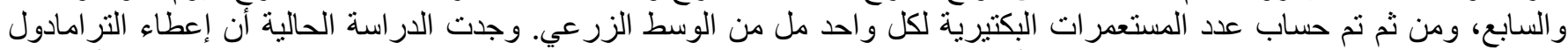

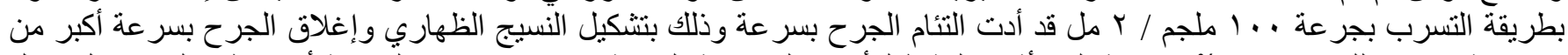

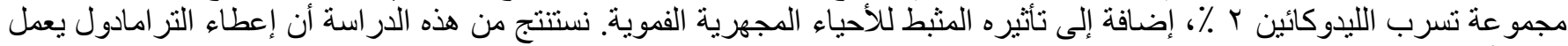

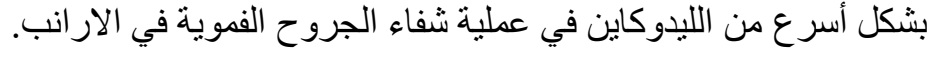




\section{Introduction}

Analgesics and anesthetics are widely used in dental practice, effects of these drugs on wound healing is worth to be studied. In dental practice, local anesthetic must have efficiency, safety, and operative needs (1). Lidocaine has been used as a local anesthetic for more than half a century. Routinely, 2\% lidocaine hydrochloride is a standard dental anesthetic drug (1). The regulation of wound healing is depending upon specific strategies to find new molecular methods or certain mediators of this healing process, like serotonergic system including serotonin and its receptor interaction that lead to induction of signaling response. Serotonin is a neurotransmitter, that has antidepressant effects in the central nervous system. It causes many cellular responses by various serotonergic receptors (2). Tramadol $\left(\mathrm{C}_{16} \mathrm{H}_{25} \mathrm{NO}_{2}\right)$ is a synthetic analgesic agent which is a centrally acting with mechanism predominantly depend on inhibition of both serotonin reuptake and norepinephrine transporter function $(3,4)$. It can be a good alternative to local anesthetic agents and beneficial to support anesthesia during long operations (5). In addition to that, the antimicrobial effect of tramadol has been approved during laboratory evaluations (6), it has different antibacterial activities mainly against Staphylococcus and Escherichia coli (7), but its antimicrobial effect in oral cavity is still not approved.

Wound healing within the oral cavity is an extremely complex mechanism where multiple characters may intervene. It has three steps: the invasion of inflammatory cells, repairing cells proliferation, and tissue remodulations. Stages of wound healing based on many factors including size of wound, circulation of the wound area, presence of foreign bodies, age, infection, patient's health condition, nutrition, drugs, and systemic diseases. The buccal mucosal lining of the oral cavity consists of stratified squamous epithelium with underlying lamina propria (8). The mucosal line which is non-keratinized stratified squamous epithelium represent the largest part about $60 \%$ of the oral mucosa, it covers the floor of mouth, lips, checks, and soft palate (9). The aim of this study was to compare clinical and histological changes of oral mucosal wound healing in rabbits after tramadol infiltration and to compare it with lidocaine infiltration and to study the antimicrobial activity of tramadol and it is effect on oral wound healing.

\section{Materials and methods}

The study was approved and carried out in the Department of Basic Science, College of Dentistry, University of Mosul. Fifteen healthy male rabbits of 5-6 months old and body weight of $1.5 \pm 0.25 \mathrm{~kg}$ purchased from local market were included in the study. They were randomly divided into three groups. In control group A (5 animals), submucosal buccal clean wound was done after infiltration with regular saline solution; in group B (5 animals) were infiltrated with $2 \%$ lidocaine and in group $\mathrm{C}$ (5 animals) were infiltrated with tramadol (Figure1). All animals were slaughtered on day 10 and buccal wounds evaluated by histologic examination. All samples were fixed in $10 \%$ formalin, embedded in paraffin, cut into $5 \mu \mathrm{m}$ sections perpendicular to the surgical line, and stained with hematoxylin-eosin then under a light microscope these sections were examined by a specialized pathologist (8).

\section{Microbiological study}

For evaluation of tramadol antimicrobial effects, swabs were done at sites of wounds before incision and other swabs were taken after surgical incision on the first day, third day and seventh day. Each swab was placed in sterile test tube containing $5 \mathrm{ml}$ of sterile brain heart infusion broth, then $0.5 \mathrm{ml}$ of brain heart broth were taken from each tube and placed in screw-capped vials containing $4.5 \mathrm{ml}$ of sterile brain heart infusion broth. To determine the number of microorganisms in the last dilutions replicate specimens $10^{7}$ and $10^{8}, 100 \mu \mathrm{l}$ of each dilution were transferred to 2 plates of blood agar. The plates were incubated at $37 \mathrm{C}^{\circ}$ for $18-48 \mathrm{hrs}$. The colony forming units per milliliter (CFU/ ML) were then calculated (10). The differences between three study groups were statistically analyzed by using two-way analysis of variance and the Duncan test. The level of significance was at $\mathrm{P} \leq 0.05$.

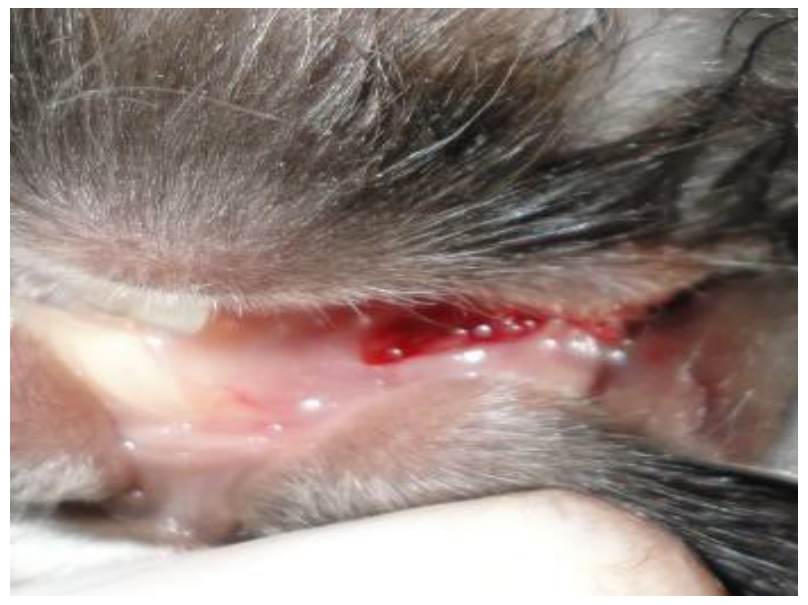

Figure 1: Photographs of oral mucosal wound in rabbit.

\section{Results}

In this study the wound healing was nearly complete after 10 days in all groups but differences in stage of healing was observed between groups, it occurred more rapidly in control and tramadol groups compared with lidocaine group (Figures 2-4). Histopathological 
examinations of the buccal wound healing in tramadol group showed similarities with the histopathological characteristics of control group. In rabbits with normal saline and tramadol infiltration, epithelialization and wound healing occur faster than lidocaine group and both groups were showed older stage of healing than that of lidocaine group which was showed collection of pus cells with delayed epithelization and healing of their oral wound. The microscopic examinations of surgical wounds supported the macroscopic findings that was seen by clinical examination.

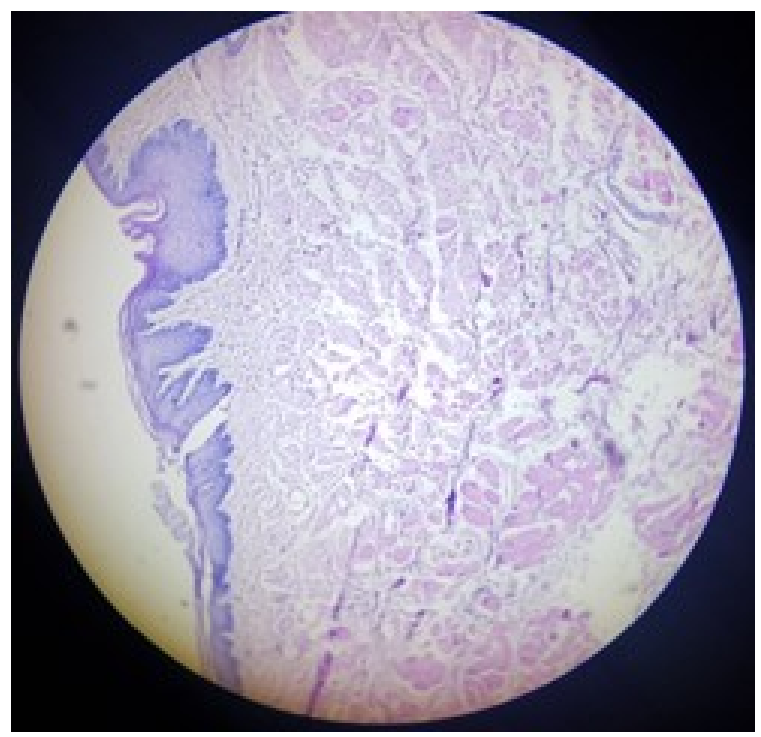

Figure 2: Microscopic healing of buccal mucosa in control group showed normal histology on day 10. H\&E stain, 40x.

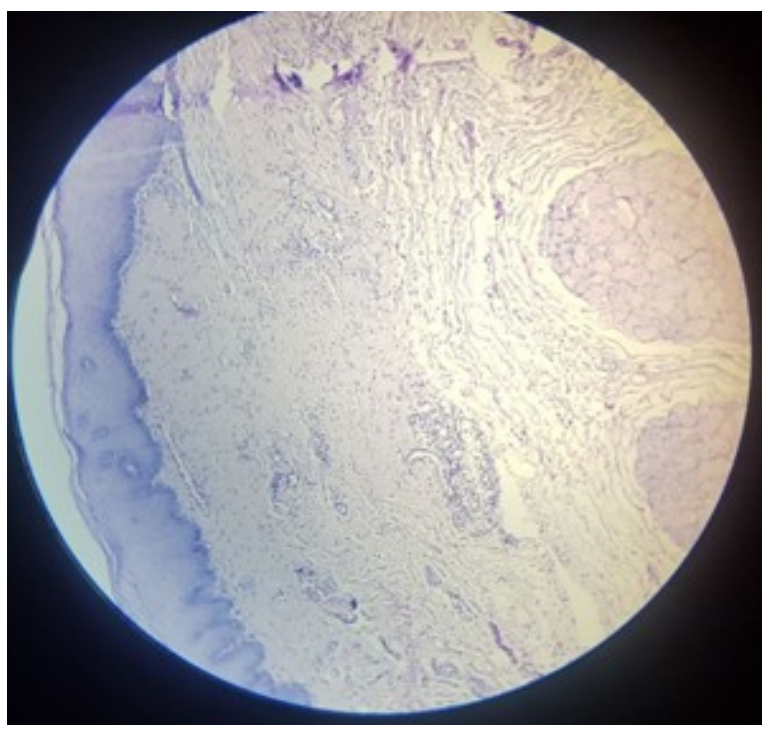

Figure 3a: Microscopic healing of buccal mucosa in tramadol group. H\&E stain, 40x.

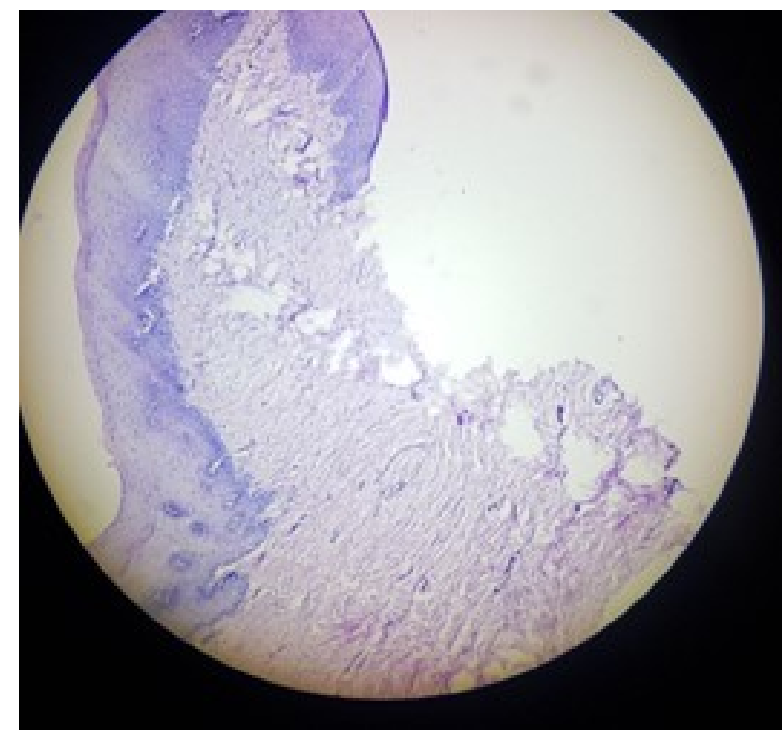

Figure 3b: Microscopic healing of buccal mucosa in tramadol group. H\&E stain, 40x.

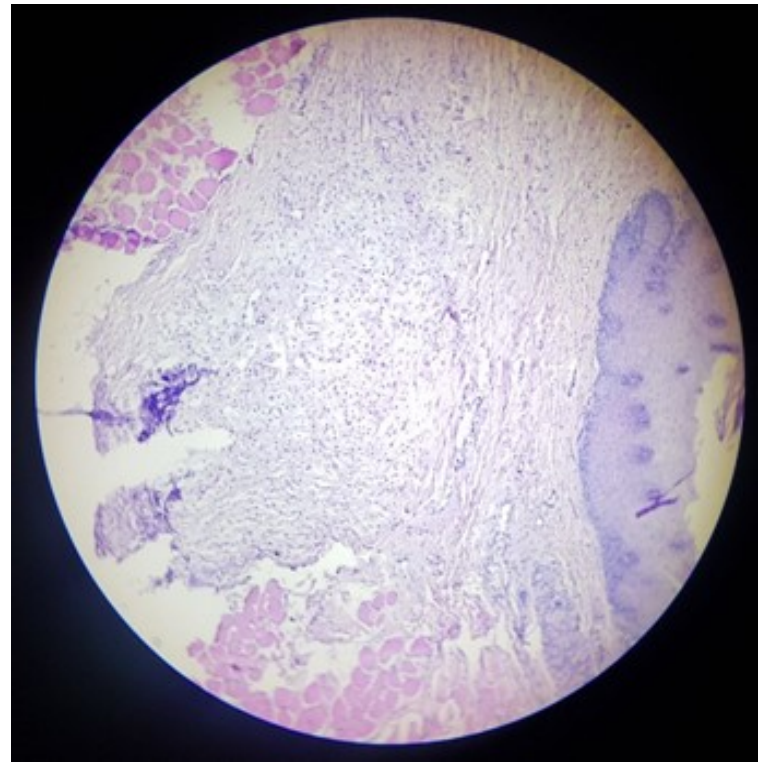

Figure 3c: Microscopic healing of buccal mucosa in tramadol group. H\&E stain, 40x.

Figure 3 a,b,c: Older stage of healing with fibrosis is more pronounced and few inflammatory cells, flat rete ridges, complete epithelization, good muscle integration in tramadol group. H\&E stain, 40x. 


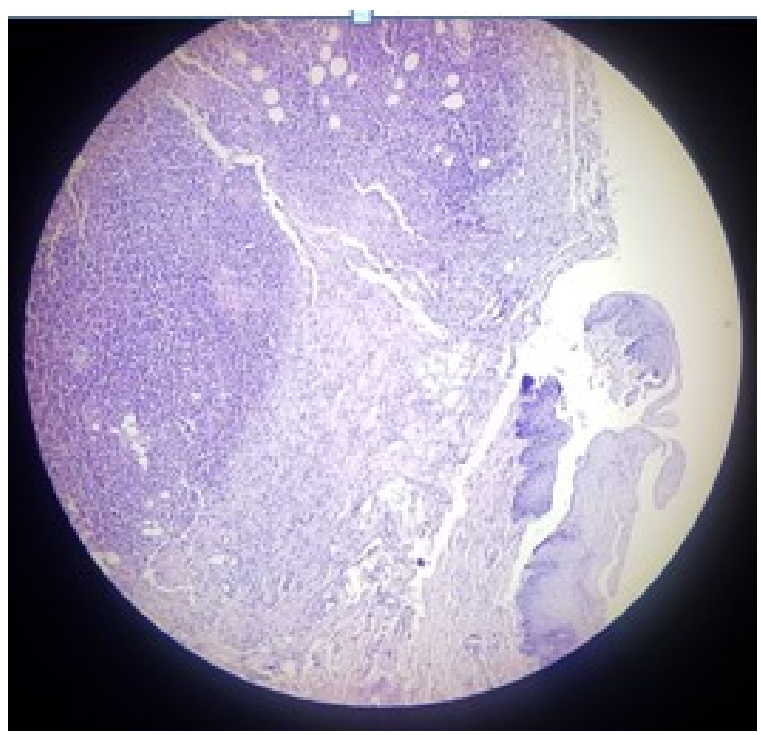

Figure 4: Microscopic healing of buccal mucosa in lidocaine group showing early stage of healing, collection of pus, no epithelization. H\&E stain, 40x.

Microbiological study results showed that tramadol and erythromycin group were significantly decrease the means of colony forming unit per milliliter (CFU) for microorganisms $77 * 10^{3} \pm 3.5$ and $64 * 10^{3} \pm 15.2$ respectively in comparison to control group $100^{*} 10^{3} \pm 18.9$ at first day. The colony forming unit per milliliter (CFU) for microorganisms at third day significantly decrease in tramadol group $52.6 * 10^{3} \pm 9.4$ and erythromycin group $39^{*} 10^{3} \pm 6.2$ at third day compared to control group $186.8^{*} 10^{3} \pm 42$. At $7^{\text {th }}$ day the $(\mathrm{CFU}) / \mathrm{ml}$ were no significant difference between tramadol and erythromycin group $10^{*} 10^{3} \pm 1.6$ and $15.2 * 10^{3} \pm 3.2$ respectively but significantly differ in comparison to control group $40 * 10^{3} \pm 12.9$.

In the present study it was found that the means of colony forming unit per milliliter $(\mathrm{CFU} / \mathrm{ml})$ for microorganisms in control was significantly increased from first day of incision $100^{*} 10^{3} \pm 18.9$ to $186.8^{*} 10^{3} \pm 42$ at third day followed by highly significantly decline to $40^{*} 10^{3} \pm 12.9$ at seventh day compared to first and third days. In erythromycin group it was found that the $(\mathrm{CFU} / \mathrm{ml})$ significantly decrease at third and seven days $39 * 10^{3} \pm 6.2$ and $15^{*} 10^{3} \pm 3.2$ respectively in comparison to first day $64 * 10^{3} \pm 15.2$. In tramadol group there was significant decrease in $(\mathrm{CFU}) / \mathrm{ml}$ after third and seventh days $52.6^{*} 10^{3} \pm 9.4$ and $10^{*} 10^{3} \pm 1.6$ respectively compared to first day $77.8^{*} 10^{3} \pm 3.5$ (Table 1 ).

Table 1: Means of colony forming unit per milliliter (CFU) for microorganisms in control negative group (normal saline), control positive group (erythromycin), and tramadol group

\begin{tabular}{lccc}
\hline No. of MO (CFU)/ml & First day & Third day & Seventh day \\
\hline Normal saline & $100^{*} 10^{3} \pm 18.9 \mathrm{~A}$ & $186.8^{*} 10^{3} \pm 42 \mathrm{~B} \mathrm{a}$ & $40^{*} 10^{3} \pm 12.9 \mathrm{C} \mathrm{a}$ \\
Antibiotic (Erythromycin) & $64^{*} 10^{3} \pm 15.2 \mathrm{~A} \mathrm{a}$ & $39 * 10^{3} \pm 6.2 \mathrm{~B} \mathrm{~b}$ & $15.2^{*} 10^{3} \pm 3.2 \mathrm{C} \mathrm{b}$ \\
Tramadol & $77.8^{*} 10^{3} \pm 3.5 \mathrm{~A} \mathrm{a}$ & $52.6^{*} 10^{3} \pm 9.4 \mathrm{~B} \mathrm{c}$ & $10^{*} 10^{3} \pm 1.6 \mathrm{C} \mathrm{b}$ \\
\hline
\end{tabular}

Data were described Mean \pm SD. Different capital letters mean there are significant different in the same raw. Different small letters mean there are significant different in the same column.

\section{Discussion}

Local anesthetics are one of the most important drugs used in dentistry, infiltration with local anesthesia to reduce postoperative pain is widely used (11). Intra oral wounds caused by surgery for treatment of pathological lesions can lead to pain, infection, and other undesirable complications like scarring and adhesion, causing functional defects, and a poor quality of life (11).

Wound healing includes a sequence of events starting by inflammation, cell migration, angiogenesis, extracellular matrix synthesis, and tissue epithelialization (12). Principally, inflammation is a protective response to remove the cause of cell injury and necrotic tissues resulting from the original insult (12). In this study histopathological examinations of the buccal wound healing in tramadol group showed similarities with the histopathological characteristics of control group., closure of wounds occurred more rapidly in control and tramadol groups than lidocaine group and both groups were showed older stage of healing than that of lidocaine group which was showed collection of pus cells with delayed healing of their oral wounds. According to results of this study tramadol infiltration at site of surgical incision appeared to help early wound healing and to reduce pus cell accumulation in oral wounds and this was in agreement with other studies which showed that wound infiltration with tramadol may be a good choice for postoperative analgesia $(13,14)$.

Wound healing is related with histopathological changes including epithelization, fibrosis, and collagen synthesis which play an important role in the maintenance of wound integrity, therefore in the present study the effect of tramadol infiltration on buccal wound healing was 
evaluated according to the histological changes, which provides information about stage of wound healing.

The function of neuroendocrine system and pain fibers are necessary factor of the wound healing, therefore infiltration of some agents like local anesthetics on the area of wound, may reduce inflammation and delay healing. According to that, the effects of lidocaine, which is a common local anesthetic in dental practice, on wound healing was studied, and different results were recorded. Some studies showed that local anesthesia reduce inflammatory response resulting in adverse effects on wound healing (15). Others reported positive effects as a consequence of reduced risk of wound infection due to the antibacterial effect of local anesthetics. The effect of opioids on wound healing is not exactly known, especially in regard to the action of opioid receptors in reducing the excitability in primary afferent neurons, which can suppress pro-inflammatory peptides such as substance $\mathrm{P}$ (13). Clark et al. showed that infiltration of morphine changed the response on wound healing by reducing cytokines and neutrophil infiltration (16). These changes could affect the healing process of wounds.

Serotonin stimulation is known to have an important role in hemostasis and tissue healing. Serotonin's regenerative and fibroproliferative effects has been documented in tissues of different organ systems (2). Considering the fact that the pharmacological effects of tramadol are associated with inhibition of reuptake of serotonin, which result in increased extracellular serotonin and could increase local serotonergic effects leading to a profound inflammatory phase and accelerate wound healing process. The positive results of tramadol on healing of oral mucosal wound was predictable given the characteristic features of this drug and its mechanism of action compared to other opioids.

According to results of this study, lidocaine group was showed delayed healing of their oral wounds suggesting that local infiltration of lidocaine produces significant histopathologic changes that substantially alter wound healing process. This was in agreement with other studies designed to evaluate the effect of lidocaine on wound healing reported by Drucker et al., Mohammad et al., Volkan et al. and Ege et al. (17-20) and disagreement of others El-Feky et al. and Kesici et al. $(12,21)$.

Some local anesthetic agent has been reported to have antibacterial effects against microorganisms that are commonly present in human skin and implicated in nosocomial infection, these local anesthetic like bupivacaine and prilocaine have bacterial activity against $E$. coli and S. epidermis (7). The antibacterial effects of local anesthetics and other agents used in wound infiltration are important.

The antibacterial properties of tramadol have been evaluated by other studies. Tramadol exhibits dose and time dependent bactericidal activity for $E$. coli and $S$. epidermidis, and bacteriostatic activity against $S$. aureus and $P$. aeruginosa strains (22). Researchers showed that tramadol may be useful in reducing bacterial infection risk after local and regional anesthesia due to its antibacterial properties. In this study isothere was no macroscopic infection at the area of the wound in all study animals. This is in agreement with other studies which emphasized the interaction of local anesthetic and tramadol's antibacterial effects (23). The antibacterial activity of tramadol may be due to depolarization and permeabilization of gram's negative outer cell membrane and inactivation of membrane bound enzyme in gram's positive bacteria (7).

In conclusion tramadol has beneficial effects on mucosal wound healing, therefore, it may be used during oral surgical procedures and its suggested to be used as a healing agent in different types of the wound after certain clinical trials. Further studies with other doses and concentrations are necessary for tramadol infiltration to be used clinically for oral surgical wounds.

\section{References}

1. Haas DA, Quinn CL. Local anesthetics. In: Frank D, Bart J, Angelo M. Pharmacology and therapeutics in dentistry. $7^{\text {th }}$ ed. New York: Mosby; 2017. 206-217 p

2. Alia S, Ahmed S, Marc GJ, Cassandra B. The role of serotonin during skin healing in post-thermal injury. Int J Mol Sci. 2018;19(4):1034. https://doi.org/10.3390/ijms19041034

3. Fayhaa AM, Ghada AT, Tahani A. Effects of tramadol on serum bone alkaline phosphatase, bone morphogenetic protein 2 and healing of dental implant in a sheep model. Intern J Pharmaceut Biol Chem Sci. 2013;2(4):13-19.www.ijpbcs.com.

4. Taqa GA. Synergism of the analgesic activities of tramadol with $\alpha 2$ adrenoreceptor agonist xylazine in mice. Iraqi $\mathrm{J}$ Vet Sci. 2012;26(2):109-113. DOI: 10.33899/ijvs.2012.67485

5. Ege B, Calisir M, Al-Haideri Y, Ege M, Gungormus M. Comparison of local anesthetic efficiency of tramadol hydrochloride and lidocaine hydrochloride. J Oral Maxillofac Surg. 2017;76(4):744-751. doi: 10.1016/j.joms.2017.11.011

6. Farzam H, Farahani A, Tafkik A, Gorgin KA, Mohajeri P, Rezaei M, Jalalvandi F. The antibacterial effect of tramadol against Staphylococcus aureus and Pseudomonas aeruginosa: In vivo study. New Microbes and New Infections. 2018;24:42-46. doi: 10.1016/j.nmni.2018.04.001

7. Al-Kurashy $\mathrm{H}$, Ismail $\mathrm{A}$, Al-windy $\mathrm{S}$. Bacteriostatic effects of tramadol. Proceeding of $3^{\text {rd }}$ scientific conference of the College of Science, University of Baghdad, 2009; 911-915 p.

8. Gupta A, Kumar P. Assessment of the histological state of the healing wound. Plast Aesthet Res.2015;2:239-42. http://www.parjournal.net.

9. Bayan JH. Effect of topical application of sesame oil on oral wound healing in rabbits. Int J Sci Res Public. 2017;7(7):885-892.

10. Benson HJ. Bacterial population counts. Microbiological applications. $8^{\text {th }}$ ed. New York: McGraw Hill; 2002. 87 p.

11. Jaewang L, Eun HK, Daiha S, Jong LR. Accelerated oral wound healing using a pre-vascularized mucosal cell sheet. Sci Rep. 2017;7(1):10667. doi: 10.1038/s41598-017-10991-x

12. 12- El-Feky GS, Abdulmaguid RF, Zayed GM, Kamel R. Mucosal codeliver of ketorolac and lidocaine using polymeric wafers for dental application. Drug Delivery. 2018;25(1):35-42. doi. Org /10. 1080/ 10717544.2017.1413445 
13. 13-Ozkan D, Seker D, Ergil J, Yalcindag A, Han U, Ginis Z, Akinci $\mathrm{M}$, Delibas N. The effects of tramadol infiltration on wound healing in rats. Acta Chir Belg. 2013;113(6):434-8. doi .org /10. 1080/ 00015458.2013.11680959.

14. Demiraran Y, Ilce Z, Kcaman B, Bozkurt P. Does tramadol wound infiltration offer an advantage over bupivacaine for postoperative analgesia in children following herniotomy? Paediatr Anaesth. 2006;16:1047-50. doi.org/10.1111/j.1460-9592.2006.01910.

15. Ferreira LM, Gragnani A, Furtado F, Hochman B. Control of the skin scarring response. An Acad Bras Cienc. 2009;81:623-629. doi.org/10.1590/S0001-37652009000300024

16. Clark JD, Shi X, Li X, Qiao Y, Liang D, Angst MS, Yeomans DC. Morphine reduces local cytokine expression and neutrophil infiltration after incision. Mol Pain. 2007;3:28. DOI: 10.1186/1744-8069-3-28.

17. Drucker M, Cardenas E, Arizti P, Valenzuela A, Gamboa A. Experimental studies on the effect of lidocaine on wound healing. World J Surg. 1998;22(4):397-8. DOI 10.1007/s002689900403.

18. Mohammad AS, Simin A, Ehsan MM. Local infiltration of tramadol versus bupivacaine for post cesarean section pain control: A doubleblind randomized study. IJMS. 2017;42(3):235. http://ijms.sums.ac.ir/article 40445.html
19. Volkan H, Sedat H, Haktan O, Sibel B, Hale S, Sükrü O, Serhan Y, Iş1l Ö. Comparison of the effects of bupivacaine, lidocaine, and tramadol infiltration on wound healing in rats. Rev Bras Anestesiol. 2012;62(6):799-810. doi: 10.1016/S0034-7094(12)70180-0.

20. Ege B, Calisir M, Al-Haideri Y, Ege M, Gungormus M. Comparison of local anesthetic efficiency of tramadol hydrochloride and lidocaine hydrochloride. J Oral Maxillofac Surg. 2018;76(4):744-751. doi: 10.1016/j.joms.2017.11.011

21. Kesici S, Kesici U, Ulusoy H, Erturkuner P, Turkmen A, Arda O. Effects of local anesthetics on wound healing. Rev Bras Anestesiol. 2018;5(17):30098-3.

http://www.scielo.br/scielo.php?script=sci issuetoc\&pid=0034$709420180005 \& \operatorname{lng}=$ en\&nrm=iso

22. Farzam H, Farahani A, Tafkik A, Gorgin A, Mohajeri P, Rezaei M, Jalalvandi F. Antibacterial effect of tramadol against Staphylococcus aureus and Pseudomonas aeruginosa: An in vivo study. New Microbes and New Infections. 2018;24:42-46. doi: 10.1016/j.nmni.2018.04.001

23. Tamanai SZ, Shacoori V, Jolivet GA, VoVan JM, Repère M, Donnio PY, Bonnaure MM. The antibacterial activity of tramadol against bacteria associated with infectious complications after local or regional anesthesia. Anesth Analg. 2007;105(2):524-527.doi: 10.1213/01.ane.0000267525.51017.b8 